\section{Clinical features and course of glutaric aciduria-Report of six cases}

Sir,

Glutaric aciduria forms a rare group of metabolic diseases with treatment options if diagnosed early. Type 1 has a prevalence of about 1 per 100,000 and characterized by accumulation of 3-OH glutaric acid and presents with hypoglycemia, vomiting, sweaty feet, chorea and failure to thrive. ${ }^{[1]}$ In Type 2 there is accumulation of 2-hydroxy glutaric acid and has a wide spectrum of presentation in clinico-demographic factors and associated with acidosis, hypoglycemia, coma, heart, liver, kidney, pancreas and skull involvement and a late form having a relatively benign course. ${ }^{[2-4]}$ Type 3 glutaric aciduria is a single peroxisomal enzyme defect causing very long-chain fatty acid deficiency and has got an entirely different clinicoradiological presentation which includes adrenoleucodystrophy and adrenomyeloneuropathies.

Patients with Type 1 and Type 2 are treated with "Well way diet" consisting of low lysine and tryptophan.

Bread, wheat and wheat products with a daily allowance of $100-150 \mathrm{kcal} / \mathrm{kg} /$ day containing low protein with a calorific value of $100-115 \mathrm{kcal} / \mathrm{kg} /$ day; protein permitted is $1-1.25 \mathrm{~g} / \mathrm{kg} /$ day, ready made preparations are available which can be used up to $350 \mathrm{mg} / \mathrm{kg} /$ day (max dose $8 \mathrm{~g} /$ day) containing l-carnitine, creatine, and glutamine:

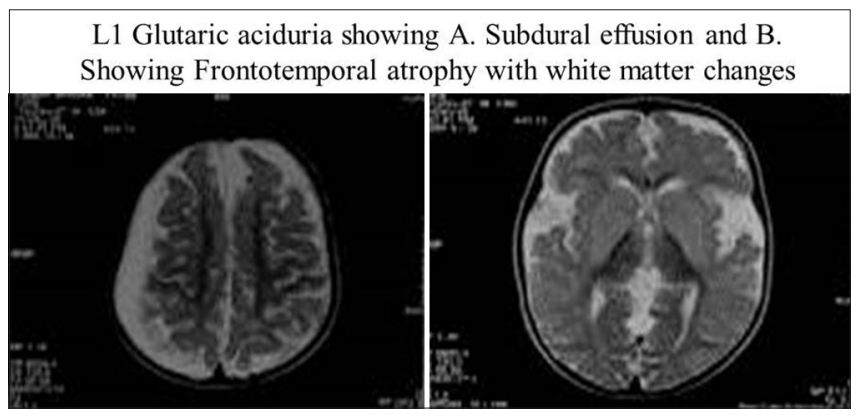

Figure 1: Open opercula, basal ganglia and white matter changes, sub-dural effusion and atrophy

L2 Glutaric aciduria - FLAIR images showing White matter and Caudate hyperintensities

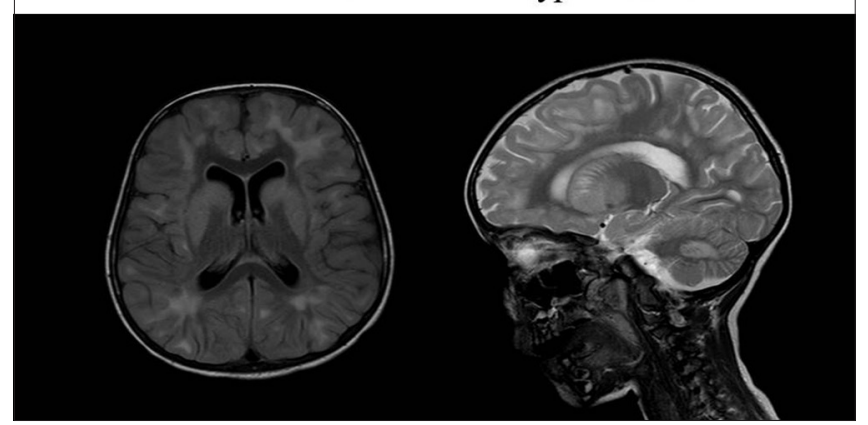

Figure 2: Periventricular white matter changes and caudate, putamen and dentate nucleus hyperintensities 
Table 1: Demographic and clinical data

\begin{tabular}{|c|c|c|c|c|c|c|c|c|c|}
\hline Diagnosis & Age & Gender & Consanguinity & $\begin{array}{l}\text { Febrile } \\
\text { seizures }\end{array}$ & $\begin{array}{l}\text { Non-febrile } \\
\text { seizures/type }\end{array}$ & Development & $\begin{array}{l}\text { Movement } \\
\text { disorders }\end{array}$ & $\begin{array}{l}\text { Pyramidal } \\
\text { signs }\end{array}$ & $\begin{array}{l}\text { Head } \\
\text { circumference }\end{array}$ \\
\hline $\begin{array}{l}\mathrm{L} 1 \\
\text { (patient 1) }\end{array}$ & 2 years & Male & Present & Present & $\begin{array}{l}\text { Present/ } \\
\text { GTCS }\end{array}$ & $\begin{array}{l}\text { Global delay with } \\
\text { acute encephalitis } \\
\text { like presentation }\end{array}$ & $\begin{array}{l}\text { Generalized } \\
\text { dystonia } \\
\text { and chorea }\end{array}$ & Absent & Normal \\
\hline $\begin{array}{l}\mathrm{L1} \\
\text { (patient 2) }\end{array}$ & 3 years & Male & Present & Absent & $\begin{array}{l}\text { Present/ } \\
\text { GTCS }\end{array}$ & Global delay & Nil & Absent & Normal \\
\hline $\begin{array}{l}\text { L2 } \\
\text { (patient 3) }\end{array}$ & 4 years & Female & Present & Present & $\begin{array}{l}\text { Present/ } \\
\text { focal motor }\end{array}$ & Normal & Nil & Absent & Normal \\
\hline $\begin{array}{l}\mathrm{L} 2 \\
\text { (patient 4) }\end{array}$ & 10 years & Male & Present & Absent & $\begin{array}{l}\text { Present/ } \\
\text { GTCS }\end{array}$ & Global delay & Ataxia & Absent & $\begin{array}{l}\text { Megalencephaly } \\
\mathrm{HC}-55.5 \mathrm{cms}\end{array}$ \\
\hline $\begin{array}{l}\mathrm{L} 2 \\
\text { (patient 5) }\end{array}$ & 28 years & Female & Present & Absent & $\begin{array}{l}\text { Present/ } \\
\text { myoclonic }\end{array}$ & Cognitive delay & $\begin{array}{l}\text { Ataxia and } \\
\text { myoclonus }\end{array}$ & Present & Normal \\
\hline $\begin{array}{l}\text { L2 } \\
\text { (patient 6) }\end{array}$ & 40 years & Female & Present & Absent & Absent & Normal & Chorea & Present & Normal \\
\hline
\end{tabular}

GTCS: Generalized tonic clonic seizure

Table 2: Other system changes including dysmorphism

\begin{tabular}{lllll}
\hline Diagnosis & Skeletal changes & Facial dysmorphism & Skin changes & $\begin{array}{l}\text { Heart, kidney and } \\
\text { liver involvement }\end{array}$ \\
\hline L1 (patient 1) & Nil & $\begin{array}{l}\text { Flat nose, low set ears, } \\
\text { inverted upper lip }\end{array}$ & Nil & Hepatomegaly \\
L1 (patient 2) & Nil & Flat nose, high arched palate & Nil & Normal \\
L2 (patient 3) & Nil & Flat nose & Hyperextensible joints & Normal \\
L2 (patient 4) & Scoliosis, dolicocephaly, & Abnormal ears, high arched & Café-au-lait spots, & Normal \\
& tilted pelvis, flat foot & palate, flat nose & hyperextensible joints & Normal \\
L2 (patient 5) & Scoliosis & Nil & Nil & Nil \\
L2 (patient 6) & Nil & Nil & & Normal \\
\hline
\end{tabular}

Table 3: Radiological features

\begin{tabular}{|c|c|c|c|c|c|}
\hline Diagnosis & $\begin{array}{l}\text { Subdural } \\
\text { effusion }\end{array}$ & $\begin{array}{l}\text { Open opercula/bat } \\
\text { wing appearance }\end{array}$ & $\begin{array}{l}\text { Cortical } \\
\text { atrophy }\end{array}$ & White matter hyperintensity & Basal ganglia changes \\
\hline $\begin{array}{l}\mathrm{L1} \\
\text { (patient 1) }\end{array}$ & Present & Present & $\begin{array}{l}\text { Frontal, parietal } \\
\text { and temporal }\end{array}$ & Present & Present \\
\hline $\begin{array}{l}\text { L1 } \\
\text { (patient 2) }\end{array}$ & Present & Present & $\begin{array}{l}\text { Frontal and } \\
\text { temporal }\end{array}$ & Present & Present \\
\hline \multirow[t]{2}{*}{$\begin{array}{l}\mathrm{L} 2 \\
\text { (patient 3) }\end{array}$} & \multirow[t]{2}{*}{ Absent } & \multirow[t]{2}{*}{ Absent } & \multirow[t]{2}{*}{ Temporal lobe } & $\begin{array}{l}\text { Predominantly around frontal and occipital } \\
\text { horn. }\end{array}$ & \multirow{2}{*}{$\begin{array}{l}\text { Swollen appearance and signal } \\
\text { changes present in caudate, } \\
\text { putamen and dentate nucleus }\end{array}$} \\
\hline & & & & $\begin{array}{l}\text { T2 and FLAIR hyper and T1 hypointense } \\
\text { signal changes. }\end{array}$ & \\
\hline $\begin{array}{l}\text { L2 } \\
\text { (patient 4) }\end{array}$ & Absent & Absent & Absent & $\begin{array}{l}\text { Diffuse subcortical white matter changes } \\
\text { including external capsule in T2 and FLAIR }\end{array}$ & $\begin{array}{l}\text { Bilateral thalamic hypointensity, } \\
\text { and dentate hyperintensity }\end{array}$ \\
\hline $\begin{array}{l}\text { L2 } \\
\text { (patient 5) }\end{array}$ & Absent & Absent & Diffuse atrophy & $\begin{array}{l}\text { Diffuse subcortical white matter changes } \\
\text { including external capsule in T2 and FLAIR }\end{array}$ & $\begin{array}{l}\text { Signal changes present in caudate, } \\
\text { putamen and dentate nucleus }\end{array}$ \\
\hline $\begin{array}{l}\mathrm{L} 2 \\
\text { (patient 6) }\end{array}$ & Absent & Absent & No atrophy & $\begin{array}{l}\text { T1 and FLAIR white matter changes } \\
\text { including } U \text { fibers }\end{array}$ & $\begin{array}{l}\text { Basal ganglia signal changes } \\
\text { present }\end{array}$ \\
\hline
\end{tabular}

Table 4: Metabolic parameters

\begin{tabular}{|c|c|c|c|c|c|c|}
\hline Diagnosis & Blood sugars & Blood gases & Bicarbonate & Serum electrolytes & Serum ammonia & Urine for abnormal metabolites \\
\hline L1 (patient 1) & Low & Normal & Normal & Normal & Normal & Normal \\
\hline L1 (patient 2) & Normal & Normal & Normal & Normal & Normal & Normal \\
\hline L2 (patient 3) & Normal & Normal & Normal & Normal & Mildly elevated & Normal \\
\hline L2 (patient 4) & Normal & Normal & Normal & Normal & Normal & Normal \\
\hline L2 (patient 5) & Normal & Normal & Normal & Normal & Normal & Normal \\
\hline L2 (patient 6) & Normal & Normal & Normal & Normal & Normal & Normal \\
\hline
\end{tabular}


Table 5: Tandem mass spectrometry

\begin{tabular}{lll}
\hline Diagnosis & Aminoacid panel & Acylcarnitine panel \\
\hline L1 (patient 1) & Normal & Free carnitine, acetyl carnitine and glutaryl carnitine elevated \\
L1 (patient 2) & Normal & Free carnitine, acetyl carnitine and glutaryl carnitine elevated \\
L2 (patient 3) & No abnormality detected & No abnormality detected \\
L2 (patient 4) & No abnormality detected & No abnormality detected \\
L2 (patient 5) & No abnormality detected & No abnormality detected \\
L2 (patient 6) & No abnormality detected & No abnormality detected \\
\hline
\end{tabular}

Table 6: Urine organic acid profile

\begin{tabular}{|c|c|c|c|c|c|c|}
\hline Compounds & L1 (patient 1) & L1 (patient 2) & L2 (patient 3) & L2 (patient 4) & L2 (patient 5) & L2 (patient 6) \\
\hline Oxalic-2 & Not done & Not done & Insignificant elevation & 1.8-fold elevated & Insignificant elevation & Insignificant elevation \\
\hline $\begin{array}{l}\text { Valproic acid } \\
\text { (VPA)-1 }\end{array}$ & Not done & Not done & $\begin{array}{l}\text { Elevated } \\
\text { (2-5-fold elevated) }\end{array}$ & Normal & & \\
\hline Isobutryl glycine-1 & Not done & Not done & Insignificant elevation & Normal & Insignificant elevation & Insignificant elevation \\
\hline $\begin{array}{l}\text { 2-Propyl, 3-OH, } \\
\text { Pentanoic acid -2 }\end{array}$ & Not done & Not done & $\begin{array}{l}\text { Elevated } \\
\text { (2-5-fold elevated) }\end{array}$ & Normal & Insignificant elevation & Insignificant elevation \\
\hline Isovaleryl glycine-1 & Not done & Not done & Insignificant elevation & Normal & Insignificant elevation & Insignificant elevation \\
\hline Thiodiglycolic-2 & Not done & Not done & Insignificant elevation & *3.8-fold elevated & Insignificant elevation & Insignificant elevation \\
\hline $\begin{array}{l}\text { 2-Propyl- } \\
\text { Hydroxyglutaric-2 }\end{array}$ & Not done & Not done & $\begin{array}{l}\text { Elevated } \\
\text { (2-5-fold elevated) }\end{array}$ & Normal & Insignificant elevation & Insignificant elevation \\
\hline Tiglylglycine-1 & Not done & Not done & Insignificant elevation & Normal & Insignificant elevation & Insignificant elevation \\
\hline $2-\mathrm{OH}$ glutaric -3 & Not done & Not done & 23.6-fold elevated & *34.8-fold elevated & Significantly elevated & Significantly elevated \\
\hline 3-OH-Phenylacetic-2 & Not done & Not done & Insignificant elevation & Normal & Insignificant elevation & Insignificant elevation \\
\hline 4-OH-benzoic-2 & Not done & Not done & Insignificant elevation & Normal & Insignificant elevation & Insignificant elevation \\
\hline 2-OH-adipic-3 & Not done & Not done & Insignificant elevation & Normal & Insignificant elevation & Insignificant elevation \\
\hline
\end{tabular}

Table 7: Urine chromatography

\begin{tabular}{ll}
\hline Diagnosis & Internal control vs. case \\
\hline L1 patient 1 & Not done \\
L1 patient 2 & Not done \\
L2 patient 3 & Significantly elevated 2-OH glutaric acid (three times elevated) \\
& 2-Ketoglutaric acid oxime (three times elevated) \\
& 2-Deoxy tetronic acid (three times elevated) \\
& Succinic acid, thiodiglycolic acid, 2-propyl, 5-OH pentanoic acid (two times elevated) \\
L2 patient 4 & Significantly elevated 2-OH glutaric acid (three times elevated) \\
L2 patient 5 & Significantly elevated 2-OH glutaric acid (two times elevated) \\
L2 patient 6 & Significantly elevated 2-OH glutaric acid (two times elevated) \\
\hline
\end{tabular}

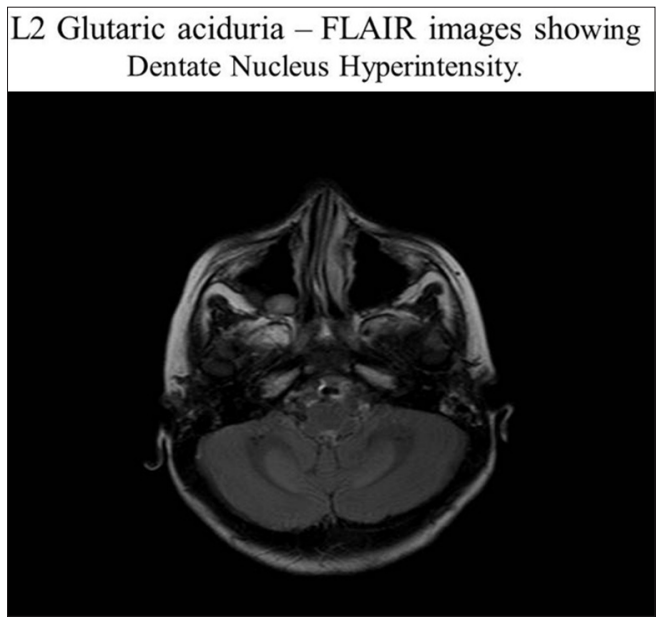

Figure 3: Dentate nucleus hyperintensities
$100 \mathrm{mg} / \mathrm{kg} /$ day each along with riboflavin and alphalipoic acid: $10 \mathrm{mg} / \mathrm{kg} /$ day each, coenzyme Q10: $8.4 \mathrm{mg} /$ $\mathrm{kg} /$ day, pantothenic acid: $5.6 \mathrm{mg} / \mathrm{kg} /$ day, alpha-linolenic acid: $150 \mathrm{mg} / \mathrm{kg} /$ day, complete pediatric vitamin: $1 / 2$ tablet daily for infants, 1 tablet for young children. Home wellday consists of phenobarbital: $4-6 \mathrm{mg} / \mathrm{kg}$-day titrated to therapeutic drug level and sick-day medications consist of extra dose with anti-inflammatory, antibiotic and antiemetics. Type 2 glutaric aciduria is treated with prevention of infection, effective maintenance of glycemic levels, supplementation of riboflavin up to $400 \mathrm{mg} /$ day, vitamin $\mathrm{C}$ and carnitine. ${ }^{[2]}$ Effective measures are taken to control inflammation, infection, dehydration, electrolyte balance and glycemic control along with managing other systemrelated co-morbidities when there is encephalopathy. We 


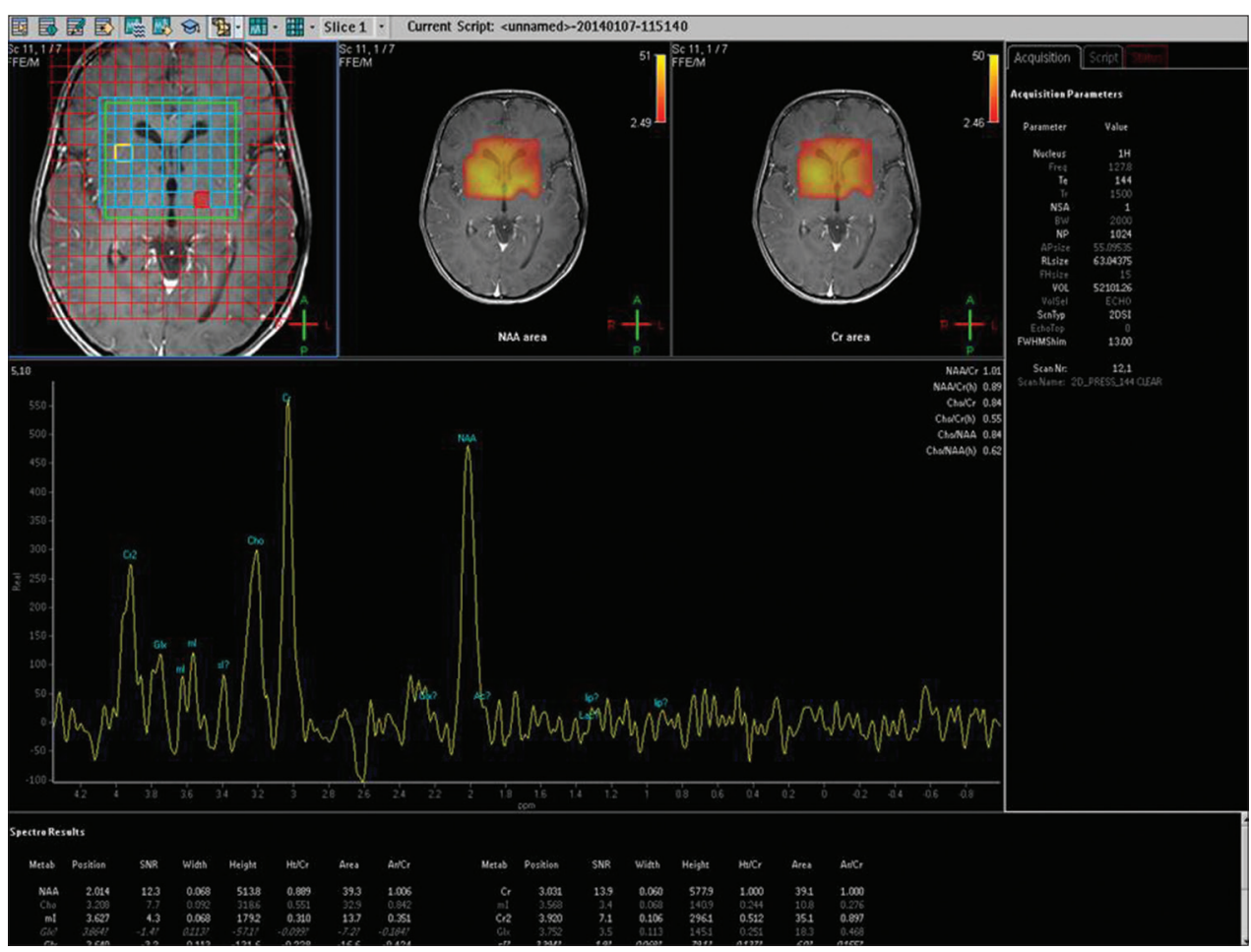

Figure 4: MRS showing small choline and no lactate peak in L2 glutaric aciduria

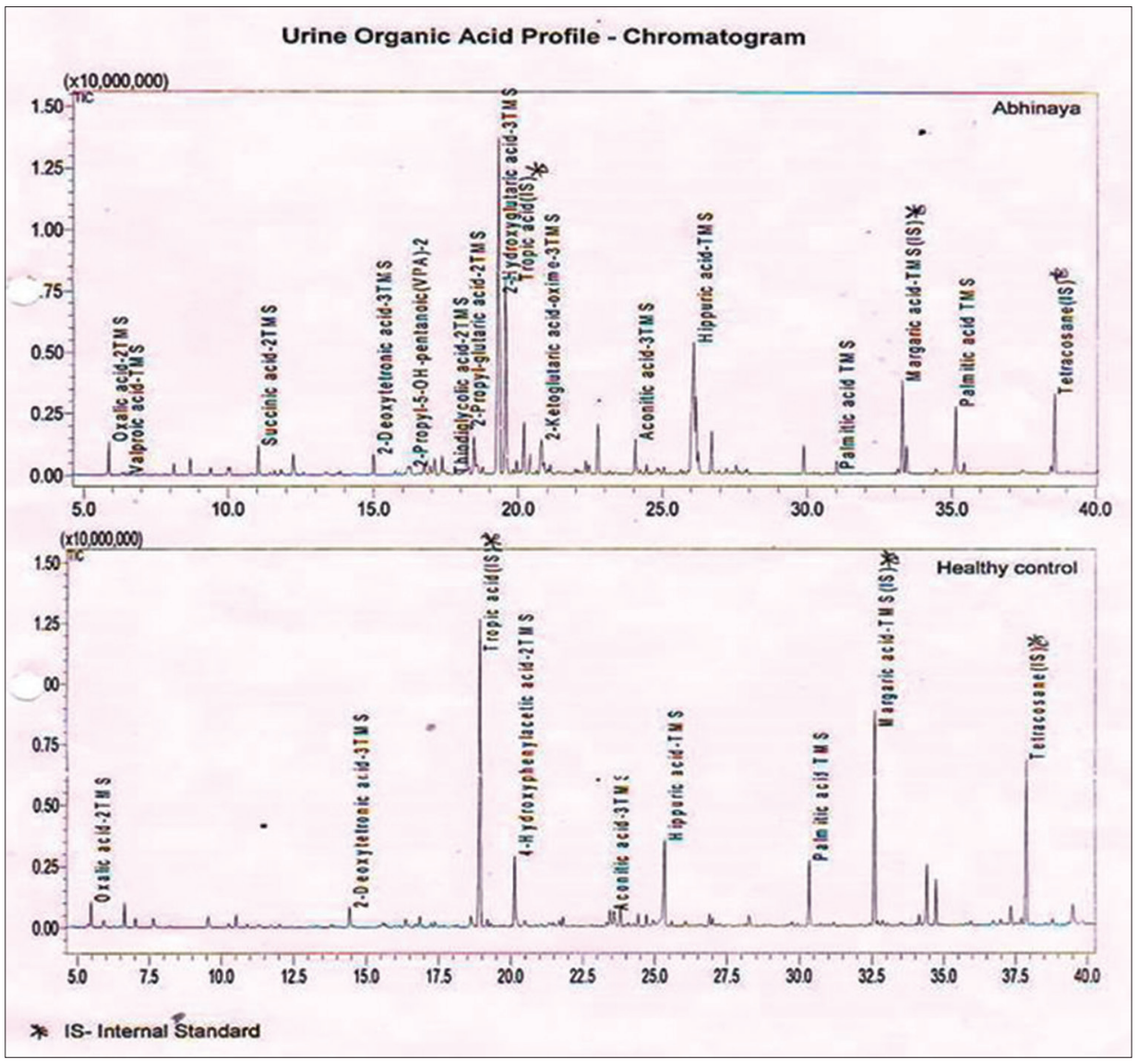

Figure 5: Urine chromatography showing 2-hydroxy glutaric acid peak 
describe two cases of L1 and four cases of L2 followed up for 3-4 years with characteristic clinical features [Tables 1 and 2], radiological features [Table 3 and Figures 1-4], tandem mass spectroscopy, urine for organic aciduria and chromatography [Tables 4-7 and Figure 5]. The study reveals that L1 glutaric aciduria presents with early, more serious neurodevelopmental and systemic complications and invariably mistaken as infective encephalopathy. L2 can show normal development and late development of seizures and cognitive impairment. ${ }^{[5,6]}$ Use of specific diet combined with disease-modifying and symptom-modifying treatment reduces morbidity greatly. They have unique radiological features, variable clinical features and are partly treatable. Therefore, a high degree of suspicion is important for diagnosis so that specific treatment can be initiated.

\section{Sadanandavalli Retnaswami Chandra, Thomas Gregor Issac', Rita Christopher ${ }^{2}$}

Department of Neurology, ${ }^{1}$ Department of Clinical Neurosciences and ${ }^{2}$ Department of Neurochemistry, National Institute of Mental Health and Neurosciences, Bangalore, Karnataka, India

Address for correspondence: Dr. Sadanandavalli Retnaswami Chandra, Faculty Block, Neurocentre, National Institute of Mental Health and Neurosciences, Bangalore - 560 029, Karnataka, India. E-mail: drchandrasasi@yahoo.com

\section{References}

1. Kamate M, Patil V, Chetal V, Darak P, Hattiholi V. Glutaric aciduria type I: A treatable neurometabolic disorder. Ann Indian Acad Neurol 2012;15:31-4.

2. Strauss KA, Puffenberger EG, Robinson DL, Morton DH. Type I glutaric aciduria, part 1: Natural history of 77 patients. Am J Med Genet C Semin Med Genet 2003;121c:38-52.

3. Genetics home reference: Available from: http://www.ghr.nlm.nih.gov/ condition/glutaric-acidemia-type-ii/show/References. [Last accessed on 2014 Sep 11].

4. Angle B, Burton BK. Risk of sudden death and acute life-threatening events in patients with glutaric acidemia type II. Mol Genet Metab 2008;93:36-9.

5. Kranendijk M, Struys EA, Salomons GS, Van der Knaap MS, Jakobs C. Progress in understanding 2-hydroxyglutaric acidurias. J Inherit Metab Dis 2012;35:571-87.

6. Patay Z, Mills JC, Löbel U, Lambert A, Sablauer A, Ellison DW. Cerebral neoplasms in L-2 hydroxyglutaric aciduria: 3 new cases and meta-analysis of literature data. AJNR Am J Neuroradiol 2012;33:940-3.

\begin{tabular}{|l|l|}
\hline \multicolumn{2}{|c|}{ Access this article online } \\
\hline Quick Response Code: & Website: \\
\hline & www.ruralneuropractice.com \\
\cline { 2 - 2 } & \\
\hline
\end{tabular}

